Supporting Information for:

\title{
Anti-biofouling Polyvinylidene Fluoride Membrane Modified by Quaternary Ammonium Compound: Direct Contact-killing versus Induced Indirect Contact-killing
}

Xingran Zhang ${ }^{1}$, Jinxing $\mathrm{Ma}^{1}$, Chuyang Y. Tang ${ }^{2}$, Zhiwei Wang*,1, How Yong $\mathrm{Ng}^{3}$, Zhichao $\mathrm{Wu}^{1}$

${ }^{1}$ State Key Laboratory of Pollution Control and Resource Reuse, School of Environmental Science and Engineering, Tongji University, 1239 Siping Road, Shanghai 200092, China

${ }^{2}$ Department of Civil Engineering, The University of Hong Kong, Pokfulam, Hong Kong, China

${ }^{3}$ Centre for Water Research, Department of Civil and Environmental Engineering, National University of Singapore, 1 Engineering Drive 2, Singapore 117576, Singapore

(*E-mail: zwwang@tongji.edu.cn, Tel./fax: +86(21)65980400)

This supporting information has 10 pages, containing four associated text sections (namely Section S1, Section S2, Section S3 and Section S4), five figures (Figure S1, Figure S2, Figure S3, Figure S4, Figure S5, Figure S6 and Figure S7), and three tables (Table S1, Table S2 and Table S3). 


\section{Contents}

Additional Details on Materials and Methods.

Section S1. Experimental procedures for intracellular ROS test.

Section S2. Details of the MBR setup.

Section S3. Procedures of qRT-PCR amplification.

Section S4. Detailed calculation procedure for XPS data.

\section{Figures}

Figure S1. A schematic illustrating the antibacterial test procedures for clean membrane coupons, membrane-soaked solution phase, pre-soaked membrane and pre-fouled membrane.

Figure S2. MBR experimental set-up.

Figure S3. (A) Roughness, (B) porosity and (C) SEM images for MP and MQ. Rq, root mean square roughness; $R \mathrm{a}$, average roughness; $R \max$, maximum roughness.

Figure S4. The $\mathrm{OD}_{600}$ value of the MP, MQ and NM (no membrane as a control) incubated for 24 h. (A) The $\mathrm{OD}_{600}$ value of E. coli upon contact with the MP and MQ [with NM (no membrane) as a control]; (B) The $\mathrm{OD}_{600}$ value of $S$. aureus upon contact with the MP and MQ [with NM (no membrane) as a control].

Figure S5. Antibacterial activity of the MP and MQ. (A) Doubling time $\left(\tau_{\mathrm{d}}\right)$ of E. coli upon contact with the MP and MQ [with NM (no membrane) as a control]; (B) $\tau_{\mathrm{d}}$ of $S$. aureus upon contact with the MP and MQ [with NM as a control].

Figure S6. CLSM images of the MQ covered with (A) alginate solution and (B) S. aureus suspension at the beginning of the incubation.

Figure S7. CLSM images of the MQ covered with (A) alginate solution and (B) S. aureus suspension at the end of the incubation.

\section{Tables}

Table S1. Detailed chemical composition for membrane preparation (unit: $\mathrm{wt} \%$ ).

Table S2. Surface elemental composition (mass \%) of membranes and pure QAC measured by XPS.

Table S3. Solids (biomass) content in the fouling layers at the end of each operation cycle in MBR. 


\section{Section S1. Experimental procedures for intracellular ROS test.}

Evaluation of QAS content in the membrane matrix. The effective membrane area is 0.5 $\mathrm{m}^{2}$, with a thickness of $250 \mu \mathrm{m}$. The quality of QAC containing in the casting solution was $0.76 \mathrm{~g}$. In theory, the concentration of QAC embedded in the membrane is $60.8 \mathrm{mg} / \mathrm{cm}^{3}$. In this section, the membrane samples were cut to pieces with a diameter of $19 \mathrm{~mm}$ (about $0.08 \mathrm{~cm}^{3}$ ). Therefore, there is $4.9 \mathrm{mg}$ QAC in each piece of membrane if no losses during the membrane preparation process occurred.

Experimental procedure of intracellular ROS test. After dipping in DI water for sufficient time, the MQ were submerged in $S$. aureus bacterial suspension (about $10^{6}$ cells $/ \mathrm{mL}$ ) which had been incubated at $37{ }^{\circ} \mathrm{C}$ for 20 min together with $\mathrm{H}_{2}$ DCF-DA. Meanwhile, identical bacterial suspension suffered from same incubation condition with $\mathrm{H}_{2}$ DCF-DA was added into $100 \mu \mathrm{L}$ pure DDBAC solution which had a far below amount of solute (5 $\mu \mathrm{g})$ compared to MQ. Finally, intracellular ROS production for both membrane and DDBAC solution was observed within several minutes. Oxidation of $\mathrm{H}_{2}$ DCF by intracellular ROS could be detected by monitoring the increase of fluorescence, which was examined at excitation $488 \mathrm{~nm}$ and emission $525 \mathrm{~nm}$ using a multi-mode microplate reader (Synergy 4, Bio-Tek Instruments Inc., America).

\section{Section S2. Details of the MBR setup}

The hydraulic retention time (HRT) and sludge retention time (SRT) of the MBR were $3.9 \mathrm{~h}$ and $30 \mathrm{~d}$, respectively. The specific aeration demand was $1.0 \mathrm{~m}^{3} /\left(\mathrm{m}^{2} \cdot \mathrm{h}\right)$. The membrane modules, each with an effective filtration area of $64 \mathrm{~cm}^{2}$, were operated at a constant permeate flux of 20 $\mathrm{L} /\left(\mathrm{m}^{2} \cdot \mathrm{h}\right)$ with an intermittent suction mode (i.e., $10 \mathrm{~min}$ suction and $2 \mathrm{~min}$ pause). The sludge concentrations of the MBR were maintained at $5.92 \pm 1.36 \mathrm{~g} / \mathrm{L}$ during the experiment. The temperature was kept constantly at $25 \pm 1{ }^{\circ} \mathrm{C}$. The TMP was recorded daily, and membrane cleaning was carried out when the TMP reached around $25 \mathrm{kPa}$.

\section{Section S3. Procedures of qRT-PCR amplification.}

The universal primer pair, i.e., Eub338F (5'-ACTCCTACGGGAGGCAGCAG-3') and Eub518R (5'-ATTACCGCGGCTGCTGG-3'), matching 16S rDNA region of bacteria were used for PCR. PCR mixture consisted of $12.5 \mu \mathrm{L}$ of $2 \mathrm{X}$ ABI Prower SybrGreen qPCR Master Mix (TaKaRa Bio Group, Japan), $0.5 \mu \mathrm{L}$ of forward primers $(10 \mu \mathrm{M})$ and $0.5 \mu \mathrm{L}$ of reverse primers $(10$ $\mu \mathrm{M}), 2 \mu \mathrm{L}$ of template cDNA, and finally the solution was adjusted to a volume of $25 \mu \mathrm{L}$ using double distilled water. The PCR protocol consisted of a hot start for $10 \mathrm{~min}$ at $95{ }^{\circ} \mathrm{C}$ followed by 40 cycles of amplification, each consisting of two steps, denaturation for $5 \mathrm{~s}$ at $95{ }^{\circ} \mathrm{C}$ and annealing/extension for $30 \mathrm{~s}$ at $60{ }^{\circ} \mathrm{C}$. Samples were also analyzed using Real-Time PCR System (ABI 7500, ABI Life Technologies, Singapore). Values were shown as the average of three measurements per membrane surface.

\section{Section S4. Detailed calculation procedure for XPS data.}

Calculation of PVDF composition at membrane surface. According to the surface elemental composition F 1s (as listed in Table S2) and molecular formula of PVDF, PVDF composition can be worked out. The results show that PVDF accounted for 76.7 and $72.4 \mathrm{wt} . \%$ of the surface compositions of MP and MQ, respectively.

Calculation of QAC composition at membrane surface. QAC-loaded membrane MQ can 
be considered as the superposition of those of MP and QAC, and the elemental balance can be expressed by the following equation, i.e., $\mathrm{E}_{\mathrm{MQ}}=\mathrm{xE}_{\mathrm{MP}}+\mathrm{y} \mathrm{E}_{\mathrm{QAC}}$, where $\mathrm{x}$ and $\mathrm{y}$ are the compositions of MP membrane and $\mathrm{QAC}$ at the membrane surface, respectively, and $\mathrm{E}_{\mathrm{MQ}}, \mathrm{E}_{\mathrm{MP}}$, and $\mathrm{E}_{\mathrm{QAC}}$ are the elemental compositions at the membrane surface as shown in Table S2. Using the above-mentioned equation and the data in Table S2, the reasonable QAC composition can be deduced using least square method. In this study, about $5.4 \mathrm{wt} . \%$ of QAC at the membrane surface was obtained.

Calculation of QAC/PVDF ratio. For the MQ coupon, the corresponding QAC to PVDF based on XPS data is equal to 5.4 wt.\% divided by PVDF composition (72.4 wt.\%), i.e., 0.075. The theoretical bulk ratio can be worked out based on the QAC dosage $(0.2 \mathrm{wt} . \%)$ and the PVDF composition ( $8 \mathrm{wt} . \%$ ) as shown in Table S1, yielding a theoretical value of 0.025 . This indicates that the QAC to PVDF mass ratio at membrane surface was 3-fold of the bulk ratio.

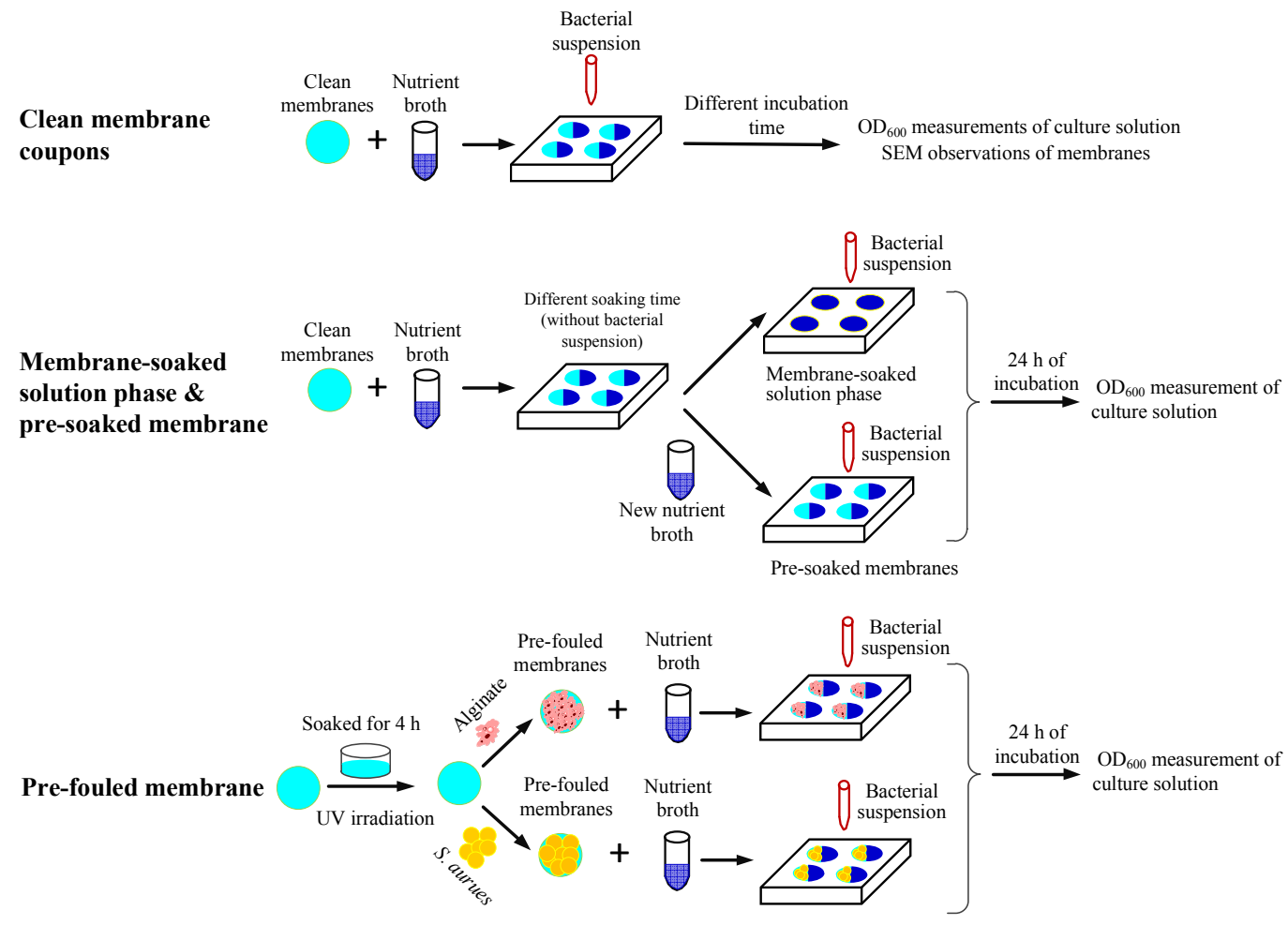

Figure S1. A schematic illustrating the antibacterial test procedures for clean membrane coupons, membrane-soaked solution phase, pre-soaked membrane and pre-fouled membrane. 


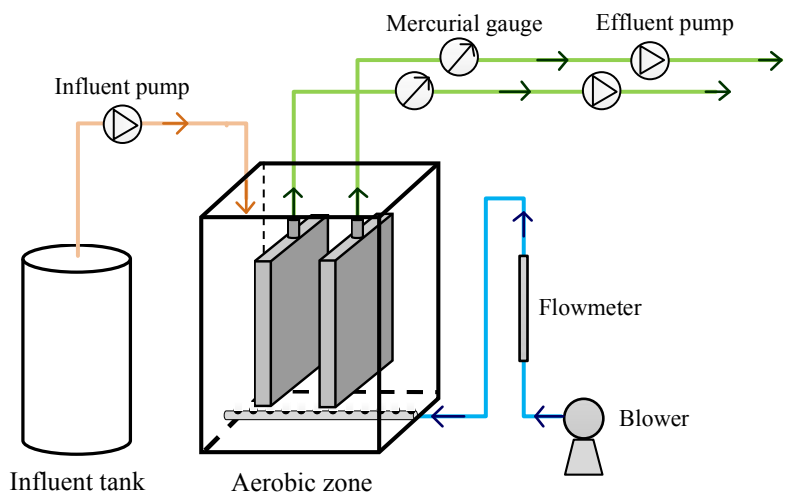

Figure S2. MBR experimental set-up.

(A)

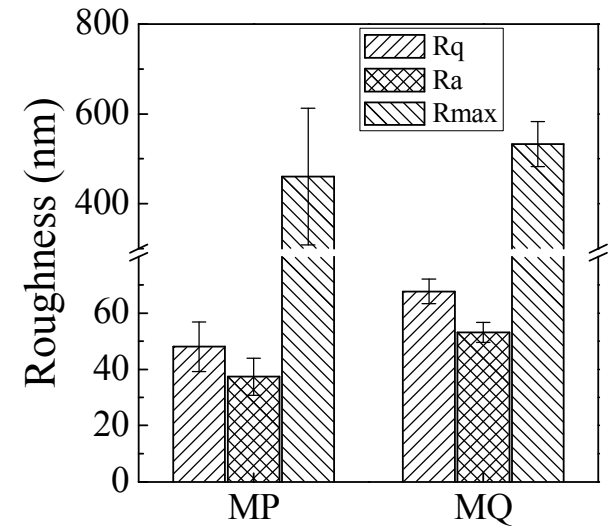

(B)

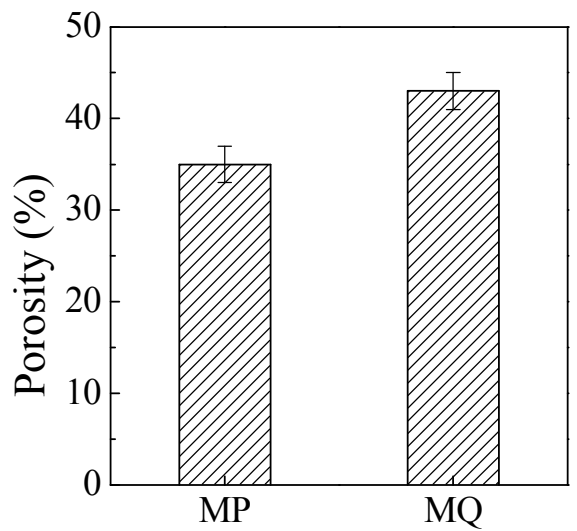

(C)

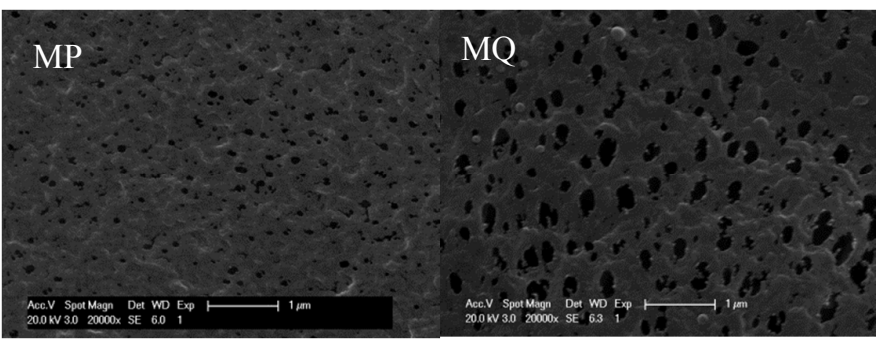

Figure S3. (A) Roughness, (B) porosity and (C) SEM images for MP and MQ. Rq, root mean square roughness; $R$ a, average roughness; $R$ max, maximum roughness. 

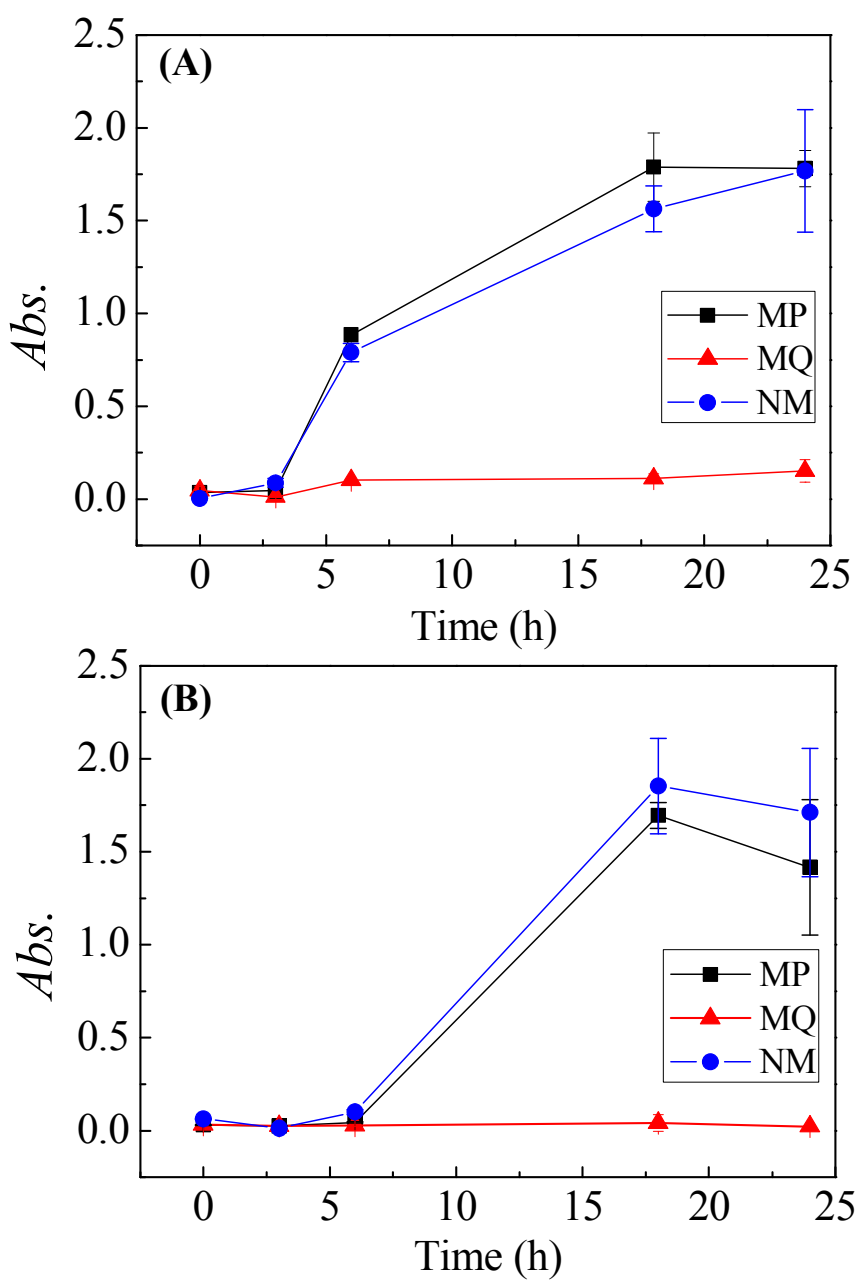

Figure S4. The $\mathrm{OD}_{600}$ value of the MP, MQ and NM (no membrane as a control) incubated for 24 h. (A) The $\mathrm{OD}_{600}$ value of $E$. coli upon contact with the MP and MQ [with NM (no membrane) as a control]; (B) The $\mathrm{OD}_{600}$ value of $S$. aureus upon contact with the MP and MQ [with NM (no membrane) as a control]. 

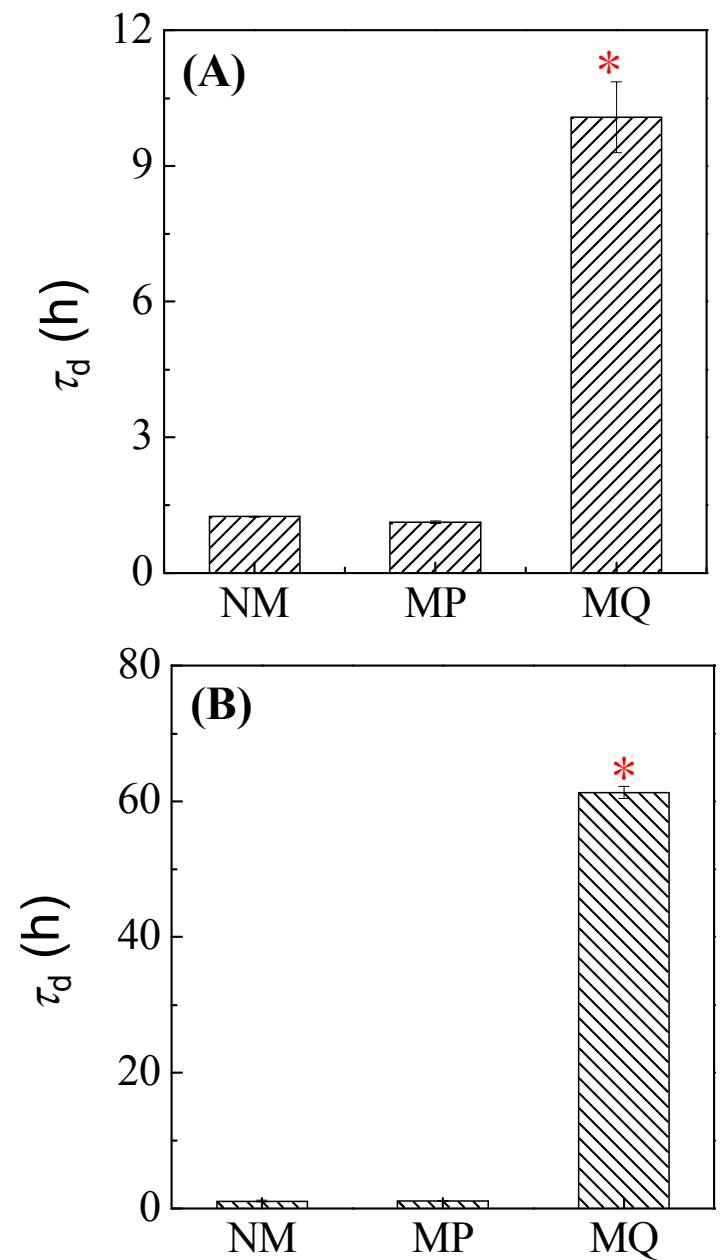

Figure S5. Antibacterial activity of the MP and MQ. (A) Doubling time $\left(\tau_{\mathrm{d}}\right)$ of $E$. coli upon contact with the MP and MQ [with NM (no membrane) as a control]; (B) $\tau_{\mathrm{d}}$ of $S$. aureus upon contact with the MP and MQ [with NM as a control]. 

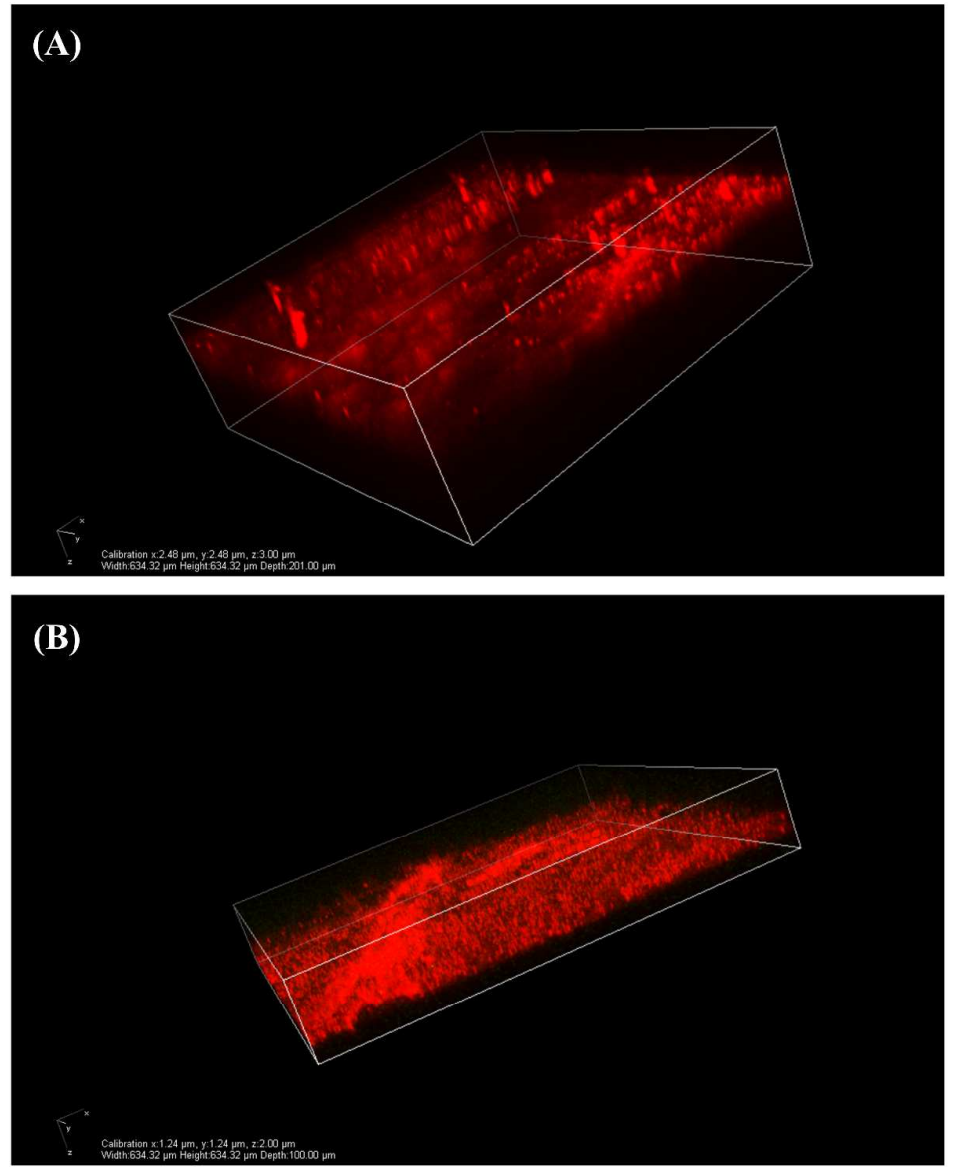

Figure S6. CLSM images of the MQ covered with (A) alginate solution and (B) S. aureus suspension at the beginning of the incubation. 

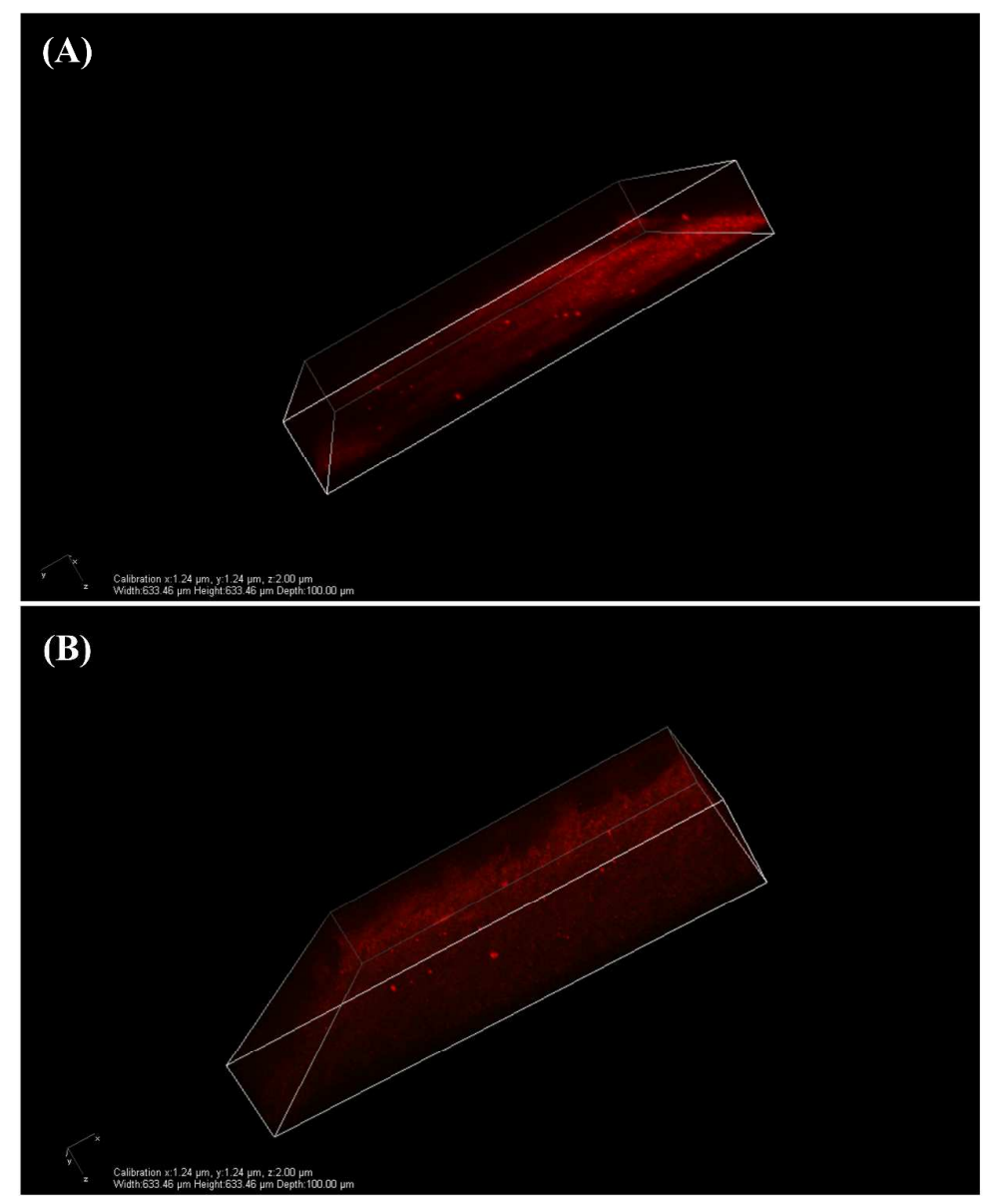

Figure S7. CLSM images of the MQ covered with (A) alginate solution and (B) S. aureus suspension at the end of the incubation.

Table S1. Detailed chemical composition for membrane preparation (unit: wt $\%$ ).

\begin{tabular}{|c|c|c|c|c|}
\hline Membranes & $\mathrm{PVDF}^{\mathrm{a}}$ & Solvent (DMAC and DMSO) ${ }^{\mathrm{a}}$ & $\mathrm{PVP}^{\mathrm{a}}$ & QAC (DDBAC $)^{\mathrm{a}}$ \\
\hline MP & 8.0 & 89.0 & 3.0 & 0.0 \\
\hline $\mathrm{MQ}^{\mathrm{b}}$ & 8.0 & 88.8 & 3.0 & 0.2 \\
\hline \multicolumn{5}{|c|}{$\begin{array}{l}\text { a. PVDF represents poly(vinylideneflouride); DMAC and DMSO denote dimethyl acetamide and } \\
\text { dimethysulfoxide, respectively; PVP indicates polyvinyl pyrrolidone; QAC and DDBAC represent quaternary } \\
\text { ammonium compound and dodecyl dimethyl benzyl ammonium chloride, respectively. b. The theoretical value of } \\
\text { the ratio of QAC amount to PVDF for MQ is } 2.5 \% \text { if QACs are evenly distributed in the PVDF matrix after phase } \\
\text { inversion (see Section S4). }\end{array}$} \\
\hline
\end{tabular}


Table S2. Surface elemental composition (mass \%) of membranes and pure QAC measured by XPS.

\begin{tabular}{ccccc}
\hline & \multicolumn{4}{c}{ Surface elemental composition } \\
\cline { 2 - 5 } & F 1s & O 1s & N 1s & C 1s \\
\hline QAC & 0 & 5.57 & 3.37 & 50.96 \\
MP & 47.03 & 4.3 & 1.76 & 46.91 \\
MQ & 44.41 & 5 & 2.39 & 48.2 \\
\hline
\end{tabular}

Table S3. Solids (biomass) content in the fouling layers at the end of each operation cycle.

\begin{tabular}{|c|c|c|c|c|}
\hline \multirow{2}{*}{$\begin{array}{c}\text { Membrane } \\
\text { samples }\end{array}$} & \multirow{2}{*}{$\begin{array}{l}\text { Time } \\
\text { (d) }\end{array}$} & \multicolumn{3}{|c|}{ Biomass } \\
\hline & & $\begin{array}{c}\mathrm{SS} \\
\left(\mathrm{g} / \mathrm{m}^{2}\right)\end{array}$ & $\begin{array}{c}\text { VSS } \\
\left(\mathrm{g} / \mathrm{m}^{2}\right)\end{array}$ & $\mathrm{VSS} / \mathrm{SS}$ \\
\hline \multirow{4}{*}{ MP } & 33 & 10.00 & 9.05 & 0.90 \\
\hline & 64 & 19.03 & 15.67 & 0.82 \\
\hline & 82 & 12.31 & 11.03 & 0.90 \\
\hline & 104 & 10.82 & 7.30 & 0.84 \\
\hline \multirow{2}{*}{ MQ } & 56 & 3.47 & 1.95 & 0.56 \\
\hline & 112 & 3.39 & 1.69 & 0.50 \\
\hline
\end{tabular}

